\title{
A study on 2 and 3-piecewise functions compared with some growth models
}

\author{
Mehmet Korkmaz \\ Department of Mathematics, Faculty of Sciences and Arts, Ordu University, Ordu, Turkey
}

Received: 23 October 2017, Accepted: 15 November 2017

Published online: 26 March 2018.

\begin{abstract}
For growth models, in addition to some classical growth models, general formations of 2 and 3 piecewise functions were given with their parameters. For finding a good model of the length of the tree, Eucalyptus camaldulensis Dehn., in addition to several sigmoidal functions (Logistic, Brody, Korkmaz-Uckardes, Gompertz, and Von Bertalanffy), 2 and 3-piecewise functions were given with their parameters. They are compared by using error sum of squares criteria. According to this criteria, it was seen that piecewise functions have minimum error sum of squares according to classical growth models used in this study. Especially it was seen that error sum of squares of 3-piecewise function has the most minimum value. For that reason, using of piecewise functions in addition to classical growth models in their studies on growth data was suggested to the researchers using growth models in their studies.
\end{abstract}

Keywords: Growth models, piecewise functions, Eucalyptus camaldulensis dehn.

\section{Introduction}

A model is an abstraction, or a simplified representation, of some aspect of reality (and should not be confused with the normative meaning of the word, something worthy of being imitated). The models are frequently used unconsciously, e.g. making mental models to visualize cause-effect relationships to help explain anticipate the behavior of systems. Models may be stated in verbal (e.g. a description) or material form (e.g. a scale model). A mathematical model is like a verbal model, but uses mathematical language which is more concise and less ambiguous than natural language. Computers have become indispensable as tools to assist modelling, but are not central to the process of modelling. Modelling is about making a good representation, and the computer is merely a convenient way to realize it (Vanclay 1994). Growth functions provide a mathematical summary of time course data on the growth of an organism (France et al. 1996). Growth models are generally used as growth curves found in a wide range of disciplines, such as fishery research, crop science, and biology (Zwietering et al. 1990). Since growth of living things are normally nonlinear, it is reasonable to explore the use of nonlinear growth model (Khamis et al. 2005). Growth models generally have sigmoidal form. Growth model is generally used as a single equation w.r.t. independent variable, time. In this study growth models were given by using piecewise functions. Most living matter grows with successive exponential, linear and saturation phases. Examples of quantities which follow such growth curves are the length or mass of a human, a tree, or a fish. In this study we used the length of a tree for showing and comparing the models. For finding a good model of the length of a tree, in addition to several sigmoidal functions (Logistic, Brody, Korkmaz-Uckardes, Gompertz and Von Bertalanffy), 2 and 3-piecewise functions were given with their parameters. The equation of the Logistic model (Ricker 1979) is obtained from the literature as:

$$
y=\frac{a}{1+e^{(b-c t)}}
$$

where $a, b, c$ are mathematical parameters, $y$ is growth value and $t$ is time. The equation of Brody model (Brody 1945) is obtained from the literature as:

$$
y=a\left(1-b e^{-c t}\right)
$$


where $a, b, c$ are mathematical parameters, $y$ is growth value and $t$ is time. The equation of the Korkmaz-Uckardes (KU) model (Korkmaz and Uckardes) is obtained from the literature as:

$$
y=a-((a-b) / \ln (2)) \times \ln (\exp (-c t)+1)
$$

where $a, b, c$ are mathematical parameters, $y$ is growth value and $t$ is time. The equation of Gompertz model (Gompertz $1825)$ is obtained from the literature as:

$$
y=a e^{\left(-e^{(b-c t)}\right)}
$$

where $a, b, c$ are mathematical parameters, $y$ is growth value and $t$ is time. The equation of Von Bertalanffy model (Von Bertalanffy 1957) is obtained from the literature as:

$$
y=a\left(1-b e^{-c t}\right)^{3}
$$

where $a, b, c$ are mathematical parameters, $y$ is growth value and $t$ is time.

\section{Material and methods}

\subsection{Material}

In this study the data taken from the tree, Eucalyptus camaldulensis Dehn. were used. The data were taken from the study of (Y1ldizbakan 2005).

Table 1: The data from Eucalyptus camaldulensis Dehn.

\begin{tabular}{|l|l|l|l|l|l|l|l|l|l|l|}
\hline Age (year) & 0 & 1 & 2 & 3 & 4 & 5 & 6 & 7 & 8 & 9 \\
\hline $\begin{array}{l}\text { Mean Length } \\
(\mathrm{m})\end{array}$ & 0.41 & 3.23 & 7.45 & 11.41 & 14.83 & 18.11 & 18.95 & 19.69 & 21.50 & 23.40 \\
\hline
\end{tabular}

\subsection{Methods}

In this study firstly, 2 and 3-piecewise functions were given in general form and then the results of the piecewise functions were given by using data of Table 1 .

\subsubsection{2-Piecewise Function}

The growth models have sigmoidal form. These models could be thought as two phases which are exponential (exp) and saturation (sat). This model can be expressed mathematically by

$$
F(t)=\left\{\begin{array}{l}
F_{\text {exp }}(t), t_{0} \leq t \leq t_{1} \\
F_{\text {sat }}(t), t \geq t_{1}
\end{array}\right.
$$

where $t$ is the time of the growth, $F(t)$ is the growth with respect to time, $\mathrm{t}_{0}$ is the starting time and $t_{1}$ is the transition time.

Figure 1 shows how the two phases of growth are represented mathematically by the model so that

$$
\begin{aligned}
& F_{\text {exp }}(t)=\alpha-1+e^{\beta\left(t-t_{0}\right)} \\
& F_{\text {sat }}(t)=b_{2}+b_{3}\left(1-e^{-\gamma\left(t-t_{1}\right)}\right)
\end{aligned}
$$


where

$\alpha$ represents the initial value of the growth.

$\beta$ represents the intrinsic growth rate of the exponential phase.

$\gamma$ represents the intrinsic saturation rate.

$t_{1}$ represents the transition time between the exponential and saturation phases.

$b_{2}$ represent the initial value of the saturation phase.

$b_{3}$ represent the asymptotic value subtracted by the initial value of the saturation phase.

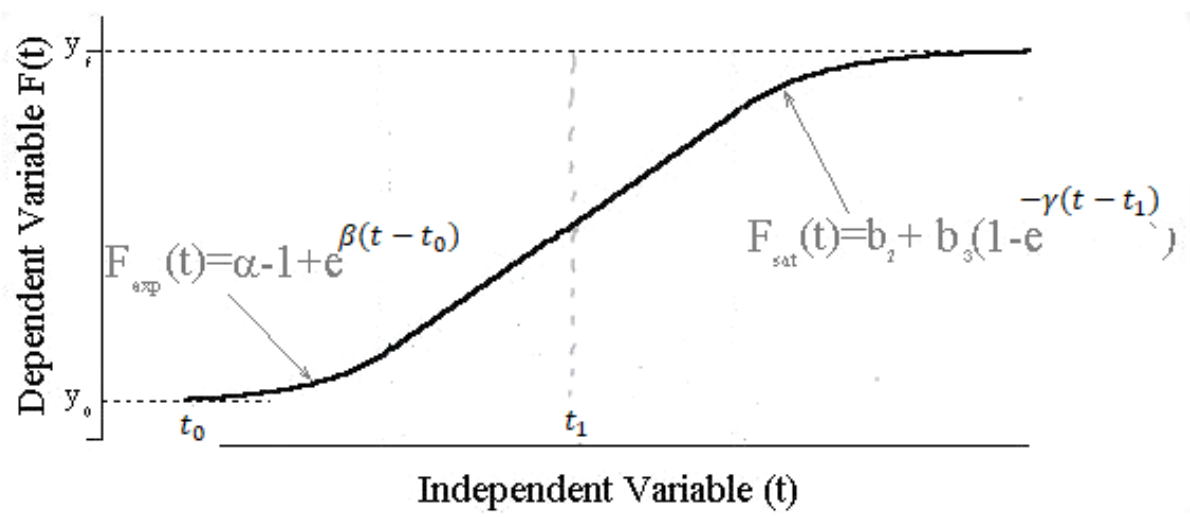

Fig. 1: The graph of the growth with two phases with respect to time.

Because of the continuity of the growth, the growth functions are the same at the transition time. In addition, their first derivatives are the same at this transition time. So we have the following equations.

$$
\begin{aligned}
& F_{\text {exp }}\left(t_{1}\right)=F_{\text {sat }}\left(t_{1}\right) \\
& f_{\text {exp }}\left(t_{1}\right)=f_{\text {sat }}\left(t_{1}\right)
\end{aligned}
$$

where $f(t)$ represents the first derivative of $F(t)$. By using the Equations 2 and 3, we get the Equations 4 and 5 , respectively.

$$
\begin{aligned}
& b_{2}=\alpha-1+e^{\beta\left(t_{1}-t_{0}\right)} \\
& b_{3}=\frac{\beta e^{\beta\left(t_{1}-t_{0}\right)}}{\gamma} .
\end{aligned}
$$

So the model in Equation 1 can be rewritten as

$$
F(t)=\left\{\begin{array}{l}
\alpha-1+e^{\beta\left(t-t_{0}\right)}, \quad t_{0} \leq t \leq t_{1} \\
\alpha-1+e^{\beta\left(t_{1}-t_{0}\right)}+\frac{\beta e^{\beta\left(t_{1}-t_{0}\right)}}{\gamma}\left(1-e^{-\gamma\left(t-t_{1}\right)}\right), \quad t \geq t_{1}
\end{array}\right.
$$




\subsubsection{3-Piecewise Function}

The growth models have sigmoidal form. These models have three phases which are exponential (exp), linear (lin) and saturation (sat). This model can be expressed mathematically by

$$
F(t)= \begin{cases}F_{\text {exp }}(t), & t_{0} \leq t \leq t_{1} \\ F_{\text {lin }}(t), & t_{1} \leq t \leq t_{2} \\ F_{\text {sat }}(t), & t \geq t_{2}\end{cases}
$$

where $t$ is the time of the growth, $F(t)$ is the growth with respect to time, $\mathrm{t}_{0}$ is the starting time and $t_{1}$ and $t_{2}$ are the transition times.

Figure 2 shows how the three phases of growth are represented mathematically by the model so that

$$
\begin{aligned}
& F_{\text {exp }}(t)=\alpha-1+e^{\beta\left(t-t_{0}\right)} \\
& F_{\text {lin }}(t)=b_{0}+b_{1}\left(t-t_{1}\right) \\
& F_{\text {sat }}(t)=b_{2}+b_{3}\left(1-e^{-\gamma\left(t-t_{2}\right)}\right)
\end{aligned}
$$

where

$\alpha$ represents the initial value of the growth,

$\beta$ represents the intrinsic growth rate of the exponential phase,

$\gamma$ represents the intrinsic saturation rate,

$t_{0}$ represents the starting time,

$t_{1}$ represents the transition time between the exponential and linear phases,

$t_{2}$ represents the transition time between the linear and saturation phases,

$b_{0}$ represent the initial value of the linear phase,

$b_{1}$ represent the rate of the linear phase,

$b_{2}$ represent the initial value of the saturation phase,

$b_{3}$ represent the asymptotic value subtracted by the initial value of the saturation phase.

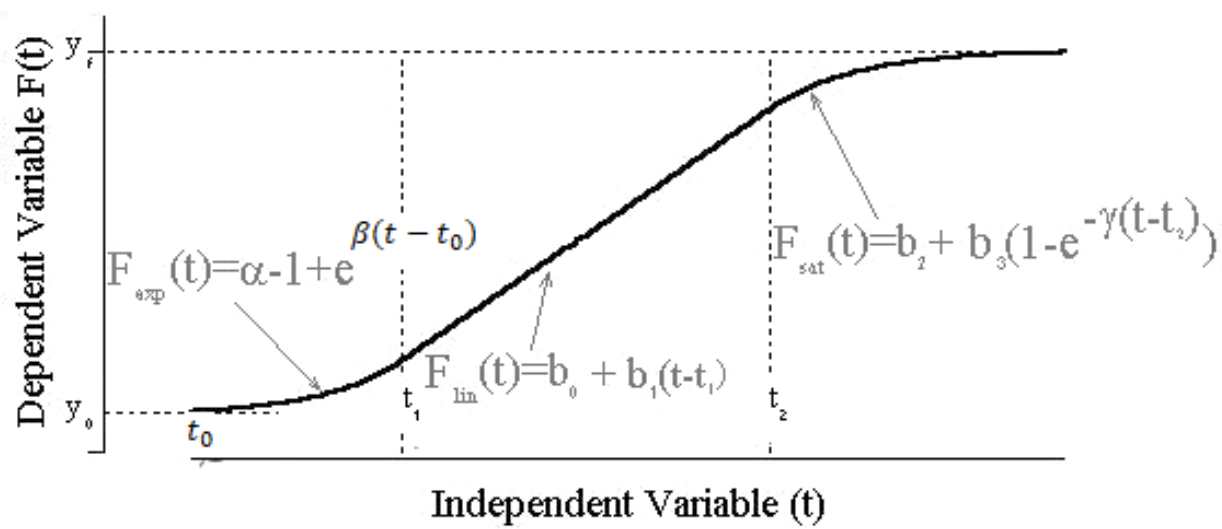

Fig. 2: The graph of the growth with three phases with respect to time. 
Because of the continuity of the growth, the growth functions are the same at the transition times. In addition, their first derivatives are the same at these transition times. So we have the following equations.

$$
\begin{aligned}
F_{\text {exp }}\left(t_{1}\right) & =F_{\text {lin }}\left(t_{1}\right) \\
F_{\text {lin }}\left(t_{2}\right) & =F_{\text {sat }}\left(t_{2}\right) \\
f_{\text {exp }}\left(t_{1}\right) & =f_{\text {lin }}\left(t_{1}\right) \\
f_{\text {lin }}\left(t_{2}\right) & =f_{\text {sat }}\left(t_{2}\right)
\end{aligned}
$$

where $f(t)$ represents the first derivative of $F(t)$. By using the Equations 7, 9, 8 and 10, we get the Equations 11, 12,13 and 14 , respectively.

$$
\begin{aligned}
& b_{0}=\alpha-1+e^{\beta\left(t_{1}-t_{0}\right)} \\
& b_{1}=\beta e^{\beta\left(t_{1}-t_{0}\right)} \\
& b_{2}=\alpha-1+e^{\beta\left(t_{1}-t_{0}\right)}+\beta e^{\beta\left(t_{1}-t_{0}\right)}\left(t_{2}-t_{1}\right) \\
& b_{3}=\frac{\beta e^{\beta\left(t_{1}-t_{0}\right)}}{\gamma}
\end{aligned}
$$

So the model in Equation 6 can be rewritten as

$$
F(t)=\left\{\begin{array}{l}
\alpha-1+e^{\beta\left(t-t_{0}\right)}, \quad t_{0} \leq t \leq t_{1} \\
\alpha-1+e^{\beta\left(t_{1}-t_{0}\right)}+\beta e^{\beta\left(t_{1}-t_{0}\right)}\left(t-t_{1}\right), \quad t_{1} \leq t \leq t_{2} \\
\alpha-1+e^{\beta\left(t_{1}-t_{0}\right)}+\beta e^{\beta\left(t_{1}-t_{0}\right)}\left(t_{2}-t_{1}\right)+\frac{\beta e^{\beta\left(t_{1}-t_{0}\right)}}{\gamma}\left(1-e^{-\gamma\left(t-t_{2}\right)}\right), \quad t \geq t_{2}
\end{array}\right.
$$

Furthermore, Table 2 shows the general form of the models used in this study.

Table 2: The general form of the models used in this study.

\begin{tabular}{|l|l|}
\hline Models & \multicolumn{1}{|c|}{$\mathrm{F}(\mathrm{t})$} \\
\hline Logistic & $\frac{a}{1+e^{(b-c t)}}$ \\
\hline Brody & $a\left(1-b e^{-c t}\right)$ \\
\hline Korkmaz-Uckardes & $a-\left(\frac{(a-b)}{\ln (2)}\right) \ln \left(e^{-c t}+1\right)$ \\
\hline Gompertz & $a e^{\left(-e^{(b-c t)}\right)}$ \\
\hline Von Bertalanffy & $a\left(1-b e^{-c t}\right)^{3}$ \\
\hline 2-Piecewise & $\left\{\begin{array}{l}\alpha-1+e^{\beta\left(t-t_{0}\right)}, t_{0} \leq t \leq t_{1} \\
\alpha-1+e^{\beta\left(t_{1}-t_{0}\right)}+\frac{\beta e^{\beta\left(t_{1}-t_{0}\right)}}{\gamma}\left(1-e^{-\gamma\left(t-t_{1}\right)}\right), t \geq t_{1}\end{array}\right.$ \\
\hline 3-Piecewise & $\left\{\begin{array}{l}\alpha-1+e^{\beta\left(t-t_{0}\right)}, t_{0} \leq t \leq t_{1} \\
\alpha-1+e^{\beta\left(t_{1}-t_{0}\right)}+\beta e^{\beta\left(t_{1}-t_{0}\right)}\left(t-t_{1}\right), t_{1} \leq t \leq t_{2} \\
\alpha-1+e^{\beta\left(t_{1}-t_{0}\right)}+\beta e^{\beta\left(t_{1}-t_{0}\right)}\left(t_{2}-t_{1}\right)+\frac{\beta e^{\beta\left(t_{1}-t_{0}\right)}}{\gamma}\left(1-e^{-\gamma\left(t-t_{2}\right)}\right), t \geq t_{2}\end{array}\right.$ \\
\hline
\end{tabular}

\section{Results and discussion}

While Table 2 shows the general form of the models used in this study, Table 3 shows the results of these models by using Table 1. According to errors sum of squares criteria, in Table 3 piecewise functions are better models with respect to the other models used in this study. Especially 3-piecewise function is the best model. The growth curves of piecewise functions and classical models used in this study are seen in the same graph (Figure 3). As shown in Figure 3, while all graphics are close to each other, the graphs of piecewise functions are quite close to each other. 
Color of Logistic, Broody, Korkmaz-Uckardes, Gompertz, Von Bertalanffy, 2 and 3-Piecewise Functions: Yellow, Black, Dark-Blue, Green, Khaki, Red and Blue, respectively.

Table 3: The results of the models used in this study.

\begin{tabular}{|c|c|c|}
\hline Models & $\mathrm{F}(\mathrm{t})$ & SSE \\
\hline \multirow[t]{2}{*}{ Logictic } & $\frac{21.881}{1+e^{(2.369-0.781 t)}}$ & \multirow[t]{2}{*}{8.713} \\
\hline & $a=21.887, b=2.369, c=0.781$ & \\
\hline \multirow{2}{*}{ Broody } & $a\left(1-b e^{-c t}\right)$ & \multirow{2}{*}{5.266} \\
\hline & $a=29.999, b=1.014, \quad c=0.166$ & \\
\hline \multirow[t]{2}{*}{ Korkmaz-Uckardes } & $a-\left(\frac{(a-b)}{\ln (2)}\right) * \ln \left(e^{-c t}+1\right)$ & \multirow[t]{2}{*}{4.700} \\
\hline & $a=27.417, b=-0.384 \quad c=0.240$ & \\
\hline \multirow{2}{*}{ Gompertz } & $a e^{\left(-e^{(b-c t)}\right)}$ & \multirow{2}{*}{3.917} \\
\hline & $a=23.071, b=1.137, \quad c=0.488$ & \\
\hline \multirow{2}{*}{ Von Bertalanffy } & $y=a\left(1-b e^{-c t}\right)^{3}$ & \multirow{2}{*}{2.875} \\
\hline & $a=23.897, b=0.711, \quad c=0.392$ & \\
\hline \multirow[t]{2}{*}{ 2-Piecewise } & $\left\{\begin{array}{l}-0.523+e^{1.255 t}, 0 \leq t \leq 1.144 \\
26.526-22.851 e^{(-0.231 t+0.264)}, t \geq 1.144\end{array}\right.$ & \multirow[t]{2}{*}{2.512} \\
\hline & $\alpha=0.477, \beta=1.255, t_{0}=0, \quad t_{1}=1.144, \gamma=0.231$ & \\
\hline \multirow[t]{2}{*}{ 3-Piecewise } & $\left\{\begin{array}{l}-0.590+e^{1.354 t}, \quad 0 \leq t \leq 0.854 \\
-1.085+4.299 t, 0.854 \leq t \leq 2.311 \\
25.802-16.952 e^{(-0.254 t+0.586)}, t \geq 2.311\end{array}\right.$ & \multirow[t]{2}{*}{2.346} \\
\hline & $\alpha=0.410, \beta=1.354, t_{0}=0, t_{1}=0.854, t_{2}=2.311, \gamma=0.254$ & \\
\hline
\end{tabular}

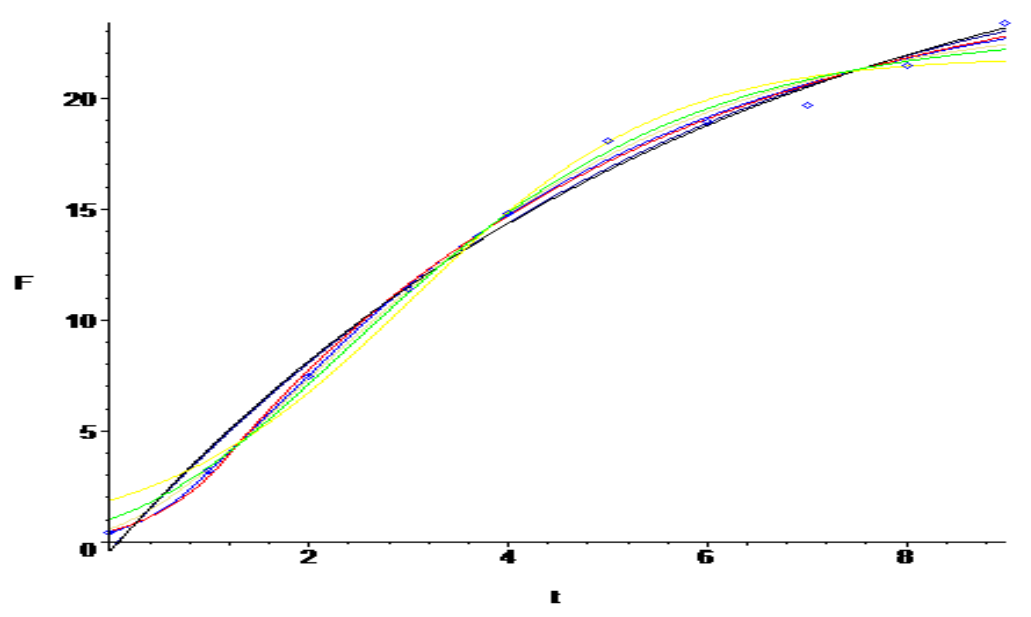

Fig. 3: The growth curves of all models used in this study.

In this study, after introducing 2 and 3-piecewise functions, we used these piecewise functions in addition to classical growth models such as Logistic, Broody, Korkmaz-Uckardes, Gompertz, Von Bertalanffy for the data on growth. We used error sum of squares criteria for comparison the models used. According to this criteria, as seen in Table 3, piecewise functions have minimum error sum of squares according to classical growth models used in this study. Especially error sum of squares of 3-piecewise function has the most minimum value. Although in this study the data taken from the tree, Eucalyptus camaldulensis Dehn. were used, these 2 and 3-piecewise functions used in this study could be used for any 
growth data. For that reason, researchers could use 2 and 3-piecewise functions in addition to classical growth models in their studies on growth data.

\section{Competing interests}

The authors declare that they have no competing interests.

\section{Authors' contributions}

All authors have contributed to all parts of the article. All authors read and approved the final manuscript.

\section{References}

[1] Brody, S. (1945). Bioenergetics and Growth. Reinhold Publishing Corporation, New York.

[2] France, J., Dijkstra, J., Dhanoa, M. S. (1996). 'Growth functions and their application in animal science', Ann. Zootech. 45, 165-174.

[3] Gompertz, B. (1825). 'On the nature of function expensive of the law of human mortality and on a new model of determining the value of life contingencies', Pphilos, Trans. K. Soc. London 115: 513-585.

[4] Khamis, A., Ismail, Z., Haron, K., Mohammed, A. T. (2005). 'Nonlinear growth Models for Modelling Oil Palm Yield Growth. Journal of Mathematics and Statistics 1 (3):225-233.

[5] Korkmaz, M., Uckardes, F. (2014). An alternative Robust model for in situ degradation studies "Korkmaz-Uckardes", Iranian Journal of Applied Animal Science 4 (1) 45-51.

[6] Ricker, W. E. (1979). 'Growth rates and models', Fish Physiol. 8: 677-743.

[7] Vanclay, J. K. (1994). Modelling Forest Growth and Yield: Applications to Mixed Tropical Forest. CAB International edition. Wallingford, UK.

[8] Von, Bertalanffy, L. (1957). 'Quantitative Laws in Metabolism and Growth', The Quarterly Review of Biology (3) 2, 218.

[9] Yildızbakan, A. (2005). Analysis on mathematical models of tree growth and comparison of these models, MSc Thesis (Turkish). University of Cukurova, Adana-Turkey.

[10] Zwietering, M. H., Jonzenburger, I., Romborts, M., Van, T., Riet, T. (1990). 'Modeling of the bacterial of growth curve', App. And Environ Microbiol 56(6), 1875-1881. 